\title{
Desarrollo humano y desigualdad de género en León, Guanajuato
}

\author{
María Áurea Valerdi González"
}

A menudo el desarrollo humano de un país se distribuye de manera desigual entre los grupos de población. Una de las posibles causas de estas disparidades hay que buscarla en la expresión

local del Índice de Desarrollo Humano (IDH) y en los indicadores que lo componen: salud, educación e ingresos. La desigualdad de género es una dimensión fundamental y a veces olvidada. Aquí mostraremos los resultados para León, San Francisco del Rincón y Purísima como zona conurbada para mostrar que aun cuando tienen un IDH medio alto, existen desigualdades socioespaciales derivadas de rezagos estructurales que no han sido atendidos. Estas localidades se suman a las "víctimas de desarrollo".

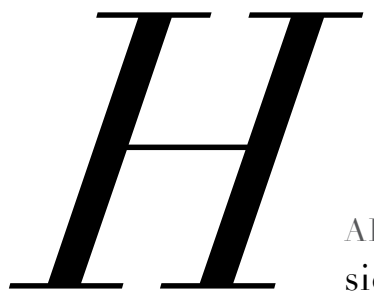

ABLAR DE DESARROLLO casi siempre nos remite a pensar en crecimiento económico, como si fueran sinónimos, medido por el producto interno bruto (PIB). Sin duda el crecimiento económico es su punto de partida pero no su única expresión. El desarrollo entendido como una meta, como un programa o un proceso supone arribar al bienestar de la población, pero el PIB no muestra el tipo de calidad de vida, pues esto im-

* Profesora-investigadora del Departamento de Estudios Sociales de la Universidad de Guanajuato, Campus León, México. plica reconocer la presencia de otros aspectos como la subjetividad, género, tiempo de trabajo, tiempo libre, planeación del estado, etcétera. Las limitantes de la medición del PIB han llevado a los organismos internacionales a exponer otras formas de reflexión sobre el desarrollo y el bienestar, como las propuestas de la Comisión Económica para América Latina (CEPAL), la Organización de las Naciones Unidas (ONU) o el Programa de las Naciones Unidas para el Desarrollo (PNUD), iniciadas en los años sesenta y setenta, cuya más reciente aportación es el índice de desarrollo humano (IDH).

El predominio del PIB como forma de medición se debe a varias razones, entre ellas a que los sistemas económicos se basan en la producción de valores de 
cambio (sobreestimados), sin contar con los valores de uso (subestimados). El PIB pondera a las personas por su ingreso y a los bienes y servicios por su precio, por eso para nadie es extraño que el PIB mida el desarrollo, porque es una cifra que se expresa con las mismas unidades que se usan en la vida cotidiana.'

El desarrollo ha pasado por diversas etapas históricas y su conceptualización teórica se ha tratado desde distintas aristas. De cómo se planifique el desarrollo depende la forma en que se concibe la inclusión o exclusión de hombres y mujeres en el mercado de trabajo.

La teoría clásica sostenía que el motor principal del crecimiento económico era la acumulación de capital fincada en el valor del trabajo, los neoclásicos rechazan esta postura y promueven la intervención del Estado poniendo una fe ciega en la lógica del mercado. Desde los años cuarenta, la CEPAL aportó nuevas categorías que vinieron a romper la concepción lineal de la corriente neoclásica: primero puso el acento en la desigualdad entre el centro y la periferia al reconocer relaciones macroeconómicas de deterioro de los mecanismos de intercambio con América Latina; segundo, formuló la teoría del modelo de industrialización por sustitución de importaciones; y tercero, sostuvo que el Estado sería la fuerza que detonaría el desarrollo. Luego cambió este principio por el enfoque dependentista, ambas posturas fueron después fuertemente criticadas. ${ }^{2}$

Para los años ochenta se contaba ya con varias concepciones distintas para hablar de desarrollo, sin superar del todo la visión economicista a nivel macro y conservando como preocupación principal la cuantificación de sus efectos. Siguiendo esta línea de pensamiento, los aportes de Amartya $\mathrm{Sen}^{3}$ significaron una nueva mirada en la comprensión del bienestar relacionado con las capacidades de los seres humanos, en especial su propuesta de instrumentos de medición, que permitieron conocer las condiciones estructurales de los países para brindar a su población los medios para su desarrollo. "Estas condiciones se articulan en torno al derecho de educación, salud, ingreso digno y el derecho a una vida prolongada y se midieron en indicadores que integran el IDH."4

La visión del desarrollo centrada en el ser humano formó parte de distintos momentos históricos y se incluyó en las agendas de los organismos internacionales como la Organización Internacional del Trabajo (OIT), que llegó a definir categorías de necesidades básicas. Esta corriente ha mostrado especial interés en la construcción de una visión integral del desarrollo que comprende además de lo económico, la dimensiones social, política, cultural y ambiental, superando así la medición centrada de manera exclusiva en el PIB, que oculta las desigualdades económicas y sociales. ${ }^{5} \mathrm{Sin}$ embargo, como señala Nazar, ${ }^{6}$ el discurso del desarrollo se ha mantenido en la esfera de la masculinidad, excluyendo a las mujeres o manteniéndolas invisibles, hasta que los movimientos feministas primero y los estudios de género después, pugnaron por su reconocimiento tanto en la producción como en la reproducción social en su sentido amplio.

Sahuí resume que en el pensamiento de Sen los funcionamientos se refieren en general a las condiciones de vida alcanzados por las personas, pero también se entienden como privación de la libertad en el sentido de "estar libre de" por ejemplo la pestes, el hambre, la desnutrición etcétera. Esta erradicación está directamente relacionada con los programas sociales y políticas públicas destinados a la población. Las capacidades, por el contrario, son visiones de futuro de lo que se podrían tener o desearían las personas, convirtiéndose así en las oportunidades que tiene un individuo de elegir entre una gama de opciones.

Si bien es cierto que el enfoque del IDH ha propiciado un acercamiento distinto al desarrollo y bienestar, no deja de ser una continuidad de los enfoques macroeconómicos cuya principal acción es medir. Por ello, una inquietud en este trabajo es ofrecer la mirada microsocial, de acercamiento a los actores, con una visión local como el territorio en el que impactan el desarrollo y bienestar, porque la integración regional también genera inequidad. El desarrollo, en este sentido, no es sino el resultado de un momento histórico del capitalismo, que sigue creando desigualdad, marginación y pobreza, derivado de la inequitativa distribución del ingreso. La idea es analizar los factores de desarrollo que impactan de manera diferenciada (hombres y mujeres), que nos permitan entender la complejidad y a su vez aproximarnos a aquellos problemas que son resultado de las crecientes asimetrías estructurales.

El modelo de desarrollo propuesto por el PNUD ha insistido en que el nivel local es el espacio más adecuado para promover procesos de beneficio social e incremento de capacidades y oportunidades para las personas. La estrategia se basa en la intervención territorial para superar y recuperar situaciones estructurales que afectan el proceso de desarrollo humano. Al territorio lo vemos como un contexto social de cooperación ac- 
tiva. La idea de construir territorios socialmente organizados, capaces de materializar iniciativas que permitan disminuir los rezagos, promover una equitativa distribución del ingreso y reducir la pobreza, no escapa a la dinámica del capital, produciendo desigualdades como veremos en el caso de León, San Francisco y Silao; ciudades que se reconfiguran y pueden potencialmente ser fuentes de conflictividad social.

\section{Polos de atracción y desarrollo en Guanajuato}

Como se ha documentado ampliamente, el IDH pretende medir tres necesidades básicas: salud, educación e ingresos. A partir de tres dimensiones esenciales:

- Índice de Sobrevivencia: La capacidad de gozar de vida larga y saludable, medida a través de la esperanza de vida al nacer.

- Índice de Educación: La capacidad de adquirir conocimientos, medida mediante una combinación del grado de alfabetismo de los adultos y el nivel de asistencia escolar, conjunto de niños, adolescentes y jóvenes_(de 6 a 24 años).

- Índice del PIB per cápita: La capacidad de contar con el acceso a los recursos que permitan disfrutar de un nivel de vida digno y decoroso medido por el PIB per cápita ajustado al poder adquisitivo del dólar estadounidense.

El IDH ha permitido a los países ubicarse en una escala que mide el nivel de desarrollo humano de sus poblaciones y propiciar, en algunos casos, nuevas acciones del Estado para cambiar su estatus. Los indicadores a nivel macro no permiten un análisis focalizado de la problemática captada que responda a las razones por las que en Guanajuato, a pesar del impulso dado al desarrollo, existen todavía rezagos estructurales e históricos.

El estado de Guanajuato presenta distintas condiciones estructurales del trabajo femenino subdividido en varios núcleos o nodos industriales como Celaya, Irapuato y León, que suponen atracción de mano de obra femenina calificada. En otras entidades federativas se ha propiciado una macrocefalia en su capital, Guanajuato en cambio es un espacio multimodal, característica poco común en México. ${ }^{8}$ El desarrollo se ha concentrado en localidades de por sí prósperas derivado de una serie de factores históricos y socioeco- nómicos, creando una fuerza centrípeta que deja fuera a otras zonas. Esta distribución de los beneficios en las ciudades también se refleja en la inserción de hombres y mujeres en el mercado de trabajo.

Bajo las actuales condiciones del mercado, el impulso dado al desarrollo industrial beneficia (con salarios directos que derivan en consumo intermedio) a las localidades y espacios que cuentan con las mejores condiciones de infraestructura y equipamiento, creando disparidades interregionales. En este contexto, hombres y mujeres de la región de estudio experimentan el impacto de procesos polarizados a partir de la creación de nodos en Guanajuato.

\section{Disparidades del DESARrollo EN lo local}

El cuestionamiento de la desigualdad de las mujeres, aportado por la perspectiva de género a los modelos de desarrollo (instrumentados desde la economía), ha enriquecido significativamente los alcances del bienestar y ha propiciado una reformulación del concepto de desarrollo. Las propuestas humanistas colocan en la mesa de discusiones el bienestar para mujeres y hombres. Con este planteamiento, se revalora el espacio privado, lo cotidiano, lo reproductivo y se reconoce la capacidad personal de las mujeres en la construcción del bienestar social. Sin embargo, la relación entre la perspectiva de género, el desarrollo y el bienestar es compleja todavía.

Con la idea de integrar elementos a la calidad de vida o bienestar, se supone que el impacto del desarrollo humano por género es crucial, pues si un grupo mejora sus indicadores y el resto de la población permanece igual, el IDH por lo general disminuye en vez de aumentar. Es decir, si se pone atención sólo al trabajo formal de hombres y mujeres (que es captado básicamente por el PIB) sin tomar en cuenta el trabajo informal, atípico, precario, flexible o vulnerable, es probable que los resultados vayan a la baja.

Se sabe que las desigualdades sociales no pueden ser capturadas por índices compuestos como el IDH, por ello se sugiere el desglose de los indicadores con el fin de identificar grupos de población vulnerables y orientar la acción política hacia cuestiones prioritarias. Algunos de los desgloses más utilizados son la dimensión espacial o geográfica, étnica, sexo, etcétera. Para el caso de la región estudiada en el Bajío de México, el desglose geográfico puede desvelar diferencias territoriales interesantes como se muestra en el cuadro I. Re- 


\begin{tabular}{|c|c|c|c|c|c|c|c|c|c|c|c|}
\hline \multirow[b]{2}{*}{ Municipios } & \multicolumn{3}{|c|}{ PEA ocupada } & \multicolumn{3}{|c|}{ PEA no ocupada } & \multicolumn{2}{|c|}{$\begin{array}{c}\text { Tasa de } \\
\text { participación }\end{array}$} & \multicolumn{3}{|c|}{ PEA por sector } \\
\hline & Total & H & M & H & M & $\begin{array}{l}\text { Labores } \\
\text { del hogar }\end{array}$ & $\mathrm{H}$ & M & Primario & Secundario & Terciario \\
\hline León & 57.8 & 76.6 & 40.2 & 23.0 & 59.5 & 52.0 & 76.6 & 40.2 & г. 6 & 43.9 & 54.4 \\
\hline Purísima & 58.2 & $79 \cdot 9$ & 37.8 & І9.6 & 6เ.9 & 58.9 & $79 \cdot 9$ & 37.8 & 14.4 & 63.7 & 2I.9 \\
\hline $\begin{array}{c}\text { San } \\
\text { Francisco }\end{array}$ & $57 \cdot 4$ & 78.6 & 38.0 & 20.9 & 6เ. 6 & 56.9 & 78.6 & 38.0 & 15.5 & 53.8 & 30.8 \\
\hline Silao & 50.8 & 74.9 & 28.7 & 24.3 & 70.9 & 58.6 & $74 \cdot 9$ & 28.7 & 20.8 & 39.I & 40.0 \\
\hline
\end{tabular}

Fuente: Panorama sociodemográfico de los municipios, INEGI, 20Io.

salta el hecho de que en Silao, 39.I por ciento de la población económicamente activa (PEA) se encuentre en el sector secundario, cuando Silao cuenta con una filial de General Motors desde 1996 y otras que se integran a la cadena de suministros.

Partimos del supuesto de que detrás del papel del Estado o la atención a las necesidades sociales de la población existe una determinada concepción del desarrollo que puede ubicarse en cualquier punto de la escala, desde la escuela neoclásica hasta los distintos enfoques posteriores como el desarrollo sustentable o el desarrollo humano. Nosotros entendemos por desarrollo aquellas acepciones que lo relacionan de modo notable con el bienestar general y con la elevación de los niveles de vida, que atraviesa por la valoración de los beneficiarios y que contempla aspectos subjetivos. Sen ha señalado que el desarrollo debe evaluarse menos con indicadores de riqueza material y más por los servicios y las oportunidades que tienen las personas, el desarrollo no es puro avance económico, sino también es político.

\section{Diferencias de GÉNERo}

Según el PNUD, ${ }^{9}$ la desigualdad de género significa que hombres y mujeres tengan las mismas oportunidades para realizar su potencial individual, para contribuir al desarrollo social y al de sí mismos. La desigualdad social que sufren las mujeres y algunos hombres no sólo es una cuestión de diferencia de sexo, como lo pensaba la economía clásica, sino una discrepancia relacionada con los modelos de producción, pues a partir del impulso dado a la industria de transformación el trabajo fuera del mercado dejó de ser considerado trabajo. A veces los problemas de género parecieran ser un asunto de mujeres, cuando en realidad es uno del orden social en su conjunto. Veamos en el siguiente cuadro el comportamiento del Índice de Diferencias de Género (IDG) para la zona de estudio.

Tanto el IDH 2005 en León como el de 20 oro en San Francisco del Rincón son considerados medio alto. Llama la atención i9 por ciento de los hogares que cuenta con jefatura femenina de Purísima del Rincón,

CuAdro 2

Comparativo del IDH e IDG en la región

\begin{tabular}{cccccc}
\hline & IDH 2005 & IDH 2010 & \% Hogar conjefatura femenina & IDG 2000 & IDG 2005 \\
\hline León & 0.8562 & 0.8355 & 23.17 & 0.8225 & 0.8487 \\
\hline Purísima & 0.8022 & 0.8707 & 19.68 & 0.7553 & 0.7924 \\
\hline San Francisco & 0.8289 & 0.9280 & 2 I & 0.7745 & 0.8204 \\
\hline Silao & 0.7846 & 0.8240 & 20.44 & 0.738 I & 0.7702 \\
\hline
\end{tabular}

Fuente: http://www.undp.org.mx/desarrollohumano/genero/Doctos/Guanajuato.pdf. (consultado el I5 de julio 20I3). El IDH 2OIO fue elaboración propia. 
pues según el cuadro i es el municipio con la PEA más alta en el sector secundario.

Desde 2000 se han incorporado otros indicadores que amplían los horizontes de análisis sobre el desarrollo humano, como el IDG, el de Empoderamiento de Género (IPG), el de pobreza (IPH-I, IPH-2), el de participación ciudadana, y otros tantos que dan cuenta de distintas problemáticas y sus diferencias territoriales. La primera edición de los IDH e IDG en México que realizó el PNUD publicada en 2006, ofreció un panorama estatal y municipal sobre el país y su vínculo con la inequidad de género. Con los resultados se hizo evidente que en general, el nivel de desarrollo de las mujeres en las entidades federativas se encuentra en desventaja frente al de los hombres. El reporte destacó que las desigualdades por razones de género son aún más pronunciadas a nivel municipal.

Como el mismo PNUD reconoce, el IDG al principio fue un indicador en etapa experimental.

El IDG no se planteó como una medida de la desigualdad de género, más bien como el IDH ajustado por las desigualdades de género en sus componentes básicos y no puede interpretarse independientemente del IDH. La diferencia entre el IDH y el IDG parece pequeña, ya que las diferencias capturadas en las tres dimensiones tienden a serlo también, dando la impresión equivocada de que las brechas de género son irrelevantes. Además, hay que calcular los ingresos disgregados por género de forma muy extrema, utilizando asunciones poco realistas debido a la falta de datos sobre ingresos diferenciados por género para más de tres cuartas partes de los países. ${ }^{10}$
De hecho en el cuadro 2 aparecen datos sobre IDG que muestran variaciones de entre 4 y 6 centésimas para el periodo 2005 y 20I0. Mientras el PNUD trabajó sobre una nueva propuesta que superaba los límites conceptuales y metodológicos que había presentado el IDG, cuyos resultados por país aparecieron en 20I3. Esta propuesta, en lugar de considerar salud, educación e ingreso, se integra por salud reproductiva (mortalidad materna y fecundidad adolescente), empoderamiento (escaños en los parlamentos y nivel de instrucción) y mercado laboral (participación en la fuerza laboral).

Muchas de las dimensiones del bienestar de las mujeres no se reflejan en las estadísticas oficiales, por eso es conveniente ir más allá de los indicadores y tener en cuenta otros elementos que expresen las particularidades de las mujeres en distintos ámbitos como la conciliación trabajo-familia, la relación trabajo productivo-reproductivo, el acceso a los servicios, ocupaciones de tiempo libre, por mencionar algunos. Sobre todo identificar de quién se está hablando, de dónde y cuándo, pues la característica de generalidad de las estadísticas no permite muchas veces cruzar en tiempo y espacio la información oficial.

Finalmente, no está por demás hacer un pronunciamiento en términos de género, pues según el PNUD" la desigualdad de género significa que hombres y mujeres tengan las mismas oportunidades para realizar su potencial individual, para contribuir al desarrollo social y al de sí mismos. Pero hacer realidad el discurso debiera atravesar por identificar las condiciones estructurales que no han permitido arribar a este propósito, ubicar en tiempo y espacio a las "víctimas del desarrollo" y atender los rezagos históricos de que son objeto.

\section{- notas $\cdot-$}

' Araceli Damián (2005), "Pobreza de tiempo en México, conceptos, métodos y situación actual”, en Mónica Gendreau (coord.) Los rostros de la pobreza. El debate, México, Iteso-Јла.

2. Estela Gutiérrez y Édgar González (2010), De las teorías del desarrollo al desarrollo sustentable, México, Siglo XXI Editores.

${ }^{3}$ Amartya Sen (20ro), La idea de justicia, México, Santillana.

${ }^{4}$ Estela Gutiérrez y Édgar González (20го), op. cit., p. II7.

${ }^{5}$ Idem.

${ }^{6}$ Austreberta Nazar y Emma Zapata (2000), "Desarrollo, bienestar y género: consideraciones teóricas”, La Ventana, núm. II, pp. 73-іI8.

7 José Sahuí (20II), "El concepto de desarrollo desde el enfoque de las necesidades humanas: una aproximación crítica" y "Capacidades y derechos: el enfoque de Martha C. Nussbaum" en José Sahuí y Antonio de la Peña (coord.), Repensar el desarrollo. Enfoques humanistas, México, Fontamara.

${ }^{8}$ Ma. Áurea Valerdi, y Juan Rodríguez (2008), "Análisis comparativo de la participación femenina en los principales nodos industriales del Bajío Guanajuatense” en Susana Suárez, Ma. Áurea Valerdi y Carlos Encinas (coords.) Dinámica localglobal: Dilemas socio-territoriales en el centro de México, Buenos Aires, Libros en Red.

9 PNUD (2OIO), Informe sobre Desarrollo Humano, http:// hdr.undp.org/es/content/informe-sobre-desarrollo-humano-20I0

${ }^{10}$ Op. cit.

"Idem. 